\title{
Geometry of contact during tooling tasks via dynamic estimation
}

\author{
Gia-Hoang Phan - Paolo Tommasino - Asif Hussain - Clint Hansen · Sylvie \\ Castagne - Domenico Campolo
}

Received: date / Accepted: date

\begin{abstract}
Contact area and contact forces between tool and workpiece are important variables for the estimation of the material removal rate during mechanical polishing, carried out during either manual or robotic operations. With a view to estimate the contact conditions during tool-workpiece interaction, a conceptual framework to capture human performance in terms of kinematics and interaction dynamics between a hand-held power tool and a workpiece is presented in this paper. In particular, a hand-held power tool was instrumented to measure 3D kinematics and 3D interaction dynamics (e.g. 3D forces and torques) during manual finishing operations. Algorithms from the literature were adapted to sense dynamically (e.g. based on force/torque measurements) the point of contact between a hard mounting-bit and the workpiece. Based on this estimation, from the shape of the mounting-bit, we estimated important geometric features. A first order analysis allows splitting contact forces into normal force and friction components. The contact point along the mounting-bit surface determines the actual relative velocity at contact and, with friction, the mechanical power involved in the finishing process. A second order analysis at contact point provides information about the curvature of the mounting-bit and the workpiece at contact. Experimental results show that the contact point between the tool and the workpiece can be precisely estimated, as validated using 3D motion capture. First and second order geometric features have been extracted from experimental data and compared with optical scans. A geometric curvature at contact can be
\end{abstract}

Gia-Hoang Phan

Robotics Research Centre, School of Mechanical and Aerospace Engineering, Nanyang Technological University, Singapore

E-mail: GIAHOANG001 @e.ntu.edu.sg

Domenico Campolo

Robotics Research Centre, School of Mechanical and Aerospace Engineering, Nanyang Technological University, Singapore

E-mail: D.CAMPOLO@ntu.edu.sg used in material removal rate models, although actual estimation of material removal is beyond the scope of this work. The results of the study are promising and based on data derived from manual operations; the proposed algorithms can be readily applied to robotic applications and used to facilitate tool path programming.

Keywords polishing $\cdot$ instrumented tool $\cdot$ robotics geometry of contact

\section{Introduction}

Surface finishing operations such as polishing and grinding are key to manufacturing and have a direct impact on the durability of the product itself [1]. Although industrial robots have been widely employed in industry, there are still many examples for which manual operations are still required, especially when accurate control of the material removal is necessary, as in the case of finesse finishing. Even when a task can be fully automated, the excessive time taken to program a robot in the traditional way (e.g. via a teaching pendant) becomes a deterrent for industries characterized by a low-volume high-mix production. An alternative is programming-by-demonstration, e.g. see [2] which is traditionally based on kinematic measurements and applied to non-contact tasks. More recently, for contact-tasks in relation to robotic assistance for disabled, force and compliance behaviour have started to be considered for the purpose of skill transfer, from human to robot [3-5]. A program by demonstration approach was successfully implemented in various tasks such as a whiteboard [6], ironing [7] and shot peening applications [8], but tool path and force trajectories could be improved if 3D interactions (kinematics and dynamics) were available during the tooling process. To automate finishing tasks, experimental methods traditionally deployed in industry are based on systematic variation of 
all process parameters and/or video examination of actual tasks carried out by skilled operators [9, 10]. Alternatively, computer simulations can be used to simulate the material removal process and the desired robotic path $[11,12]$. However, in this case, the quality of the tooling is largely dependent on the accuracy of the model (robot + material removal process) used in simulations.

For finishing operations, Material Removal Rate (MMR) determines how fast the material is being removed during surface finishing tasks and is a critical factor for process automation and tool-path programming [13]. Existing MRR models are often empirical in nature, and rely on extensive sets of prior data and regression analysis and assume pressure distributions and contact areas based on geometric factors usually derived from CAD models $[14,15]$. Hence, neglecting that especially during material removal operations, the surface and its geometry change continuously [9, 16-19].

The main objective of this work is to provide a realtime estimation, rather than an assumption, of the geometric factors behind the MRR. In particular, the objective is to characterize the geometry of contact based on dynamic estimations, i.e. estimations derived from force/torque readings, rather than just kinematics and geometric information derivable from CAD models. By fusing kinematic data (for example sensed with motion capture for manual operations or encoders in robotic operations) with force/torque sensing, our approach allows the on-line estimation of: contact point, tangent planes (first-order geometry), tangential and normal contact forces as well as curvatures (second-order geometry), a geometric factor important to estimate contact areas, e.g. in Hertzian contact models [20].

Contact estimation based on force/torque sensing was initially proposed in the 80s [21-23] and later used in different scenarios. Salisbury et al [24] described how joint torque measurements in a robot arm might be used to determine the location of a contact on its links. Bicchi et al. [25] introduced the contact centroid for characterizing soft finger contact and emphasized the role of the wrench axis [26,27], which will be described later in this work. Zhou et al. [28] formalized the method to localize the contact for robot fingers and carried out an accuracy analysis based on noise levels in force/torque sensors. Additionally, Lejun et al. [29] used a dynamic force model in robotic milling to analyse the effect of robot structural dynamics.

Unlike robotic fingers, finishing tools are subject to high levels of mechanical vibrations which introduce additional errors (mechanical noise). The contribution of this paper is 1) to adapt sensing techniques as in [28] to a finishing task, with the assumption of a rigid mounting-bit; 2) to determine, in addition to the contact point estimation, also the first and second-order geometry (i.e. tangent space and cur- vature directions) at the contact point $\left.{ }^{1} ; 3\right)$ to instrument the tool with kinematic and dynamic (force/torque) sensors; and 4) to evaluate accuracy during actual tool operation, in the presence of mechanical noise.

The paper is structured as follows: first, the contact point estimation via dynamic sensing is presented, along with the geometry of contact; then, the instrumentation of a commercial hand-held tool is presented; finally, the calibration and experimental validation during a process involving material removal are described. The outcome of the study and their potential applications are discussed in the last section.

\section{Geometry of contact via dynamic estimation}

Contact between two surfaces can be described both at a microscopic and a macroscopic level. From a robotic sensing perspective, we shall be interested in the latter and therefore rather than a distribution of micro contact forces $\boldsymbol{f}_{i}$, we shall consider a unique macro resultant force $\boldsymbol{F}=\sum \boldsymbol{f}_{i}$ applied directly at the center of pressure (CoP). The CoP can be dynamically estimated from the resultant force $\boldsymbol{F}$ and moment $\boldsymbol{T}$ as the intersection between the wrench-axis and the mounting-bit surface $S$. In the current study, we are not only interested in sensing the resultant force between the workpiece and the tool, but also in estimating the $\mathrm{CoP}$ itself (see Fig.1) by means of force/torque measurements. Although the interaction between tool and workpiece involves purely a resultant force, at the contact, a moment $\boldsymbol{T}$ can also be sensed at frames which are not co-located with the CoP. This shall always be the case since force sensors are practically always located far away from the CoP.

Considering a generic finishing tool, a convenient tool frame $\mathcal{T}$ can often be defined as having its z-direction aligned with the major axis of the mounting-bit and as being located opposite to the tooling surface. A wrench $\boldsymbol{W}$ (i.e. a combination of force and torque components $[26,27])$ can, therefore, be sensed at the tool frame $\mathcal{T}$ which can be written as:

$W=\left[\begin{array}{l}F \\ T\end{array}\right]$

where $\boldsymbol{T}$ is the torque arising at the origin of the frame $\mathcal{T}$ due to the force $\boldsymbol{F}$ applied at the CoP. In fact, the same wrench would be measured if the force $\boldsymbol{F}$ were applied at any position $\boldsymbol{r}$ along the wrench-axis [25,28], i.e. a line defined by the following relationship:

$\boldsymbol{T}=\boldsymbol{F} \times \boldsymbol{r}$

where $\times$ is the Euclidean cross product. When written in $\mathcal{T}$

1 Higher order geometry, in particular, curvature, is required to estimate material removal $[13,30]$ although the review of such models is beyond the scope of this paper 

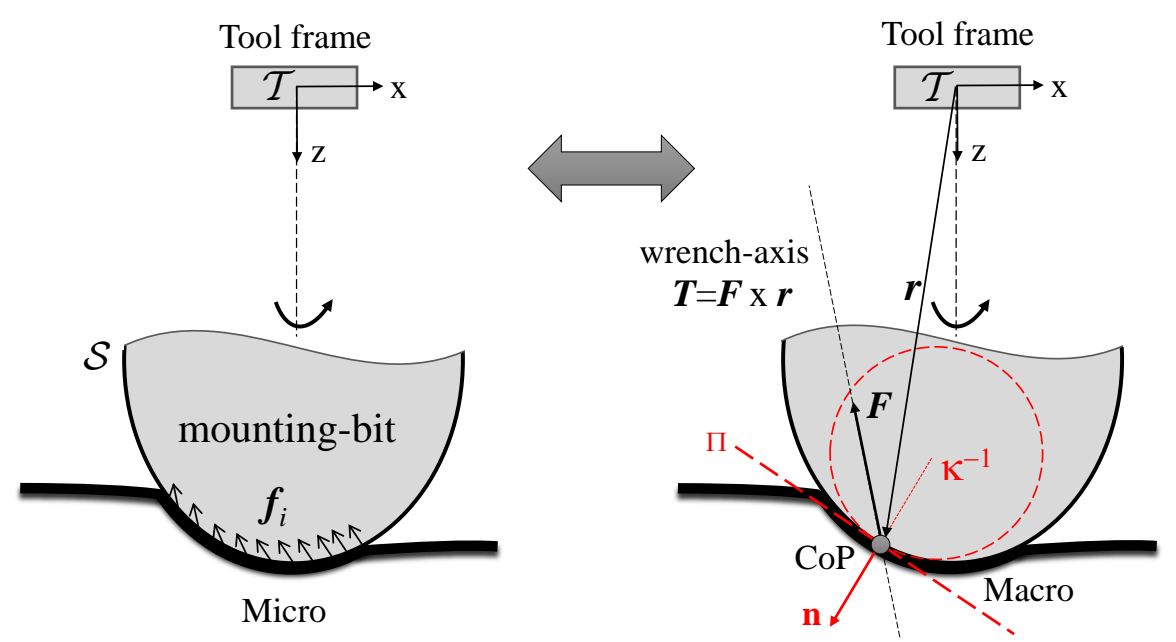

Fig. 1 Geometry of the contact between a rotating mounting-bit and a workpiece: micro forces $f_{i}$, distributed over an area (left), macro force $F$ applied at the centre of pressure (CoP) (right).

frame components, $\boldsymbol{r}=\left[\begin{array}{lll}r_{x} & r_{y} & r_{z}\end{array}\right]^{T}, \boldsymbol{F}=\left[\begin{array}{lll}F_{x} & F_{y} & F_{z}\end{array}\right]^{T}$ and $\boldsymbol{T}=\left[\begin{array}{lll}T_{x} & T_{y} & T_{z}\end{array}\right]^{T}$, Eq.(2) leads to three dependent equations $^{2}$ which define a line in 3D space:

$$
\left[\begin{array}{c}
T_{x} \\
T_{y} \\
T_{z}
\end{array}\right]=\left[\begin{array}{l}
r_{y} F_{z}-r_{z} F_{y} \\
r_{z} F_{x}-r_{x} F_{z} \\
r_{x} F_{y}-r_{y} F_{x}
\end{array}\right]
$$

In other words, the Eq.(3) consists of three equations but only two are independent, and the solutions are all the points along a line parallel to $\boldsymbol{F}$ passing through $\mathrm{CoP}$, as shown in Fig. 1 as wrench-axis.

Once the shape of the mounting-bit is known, in particular when a point $r$ on the surface $S$ can be parameterized as $\boldsymbol{r}=\left[r_{x} r_{y} S\left(r_{x}, r_{y}\right)\right]^{T}$ in $\mathcal{T}$ frame coordinates, the CoP can be computed as the intersection ${ }^{3}$ between wrench axis (3) and the surface $S$, simply represented as:

$$
r_{z}=S\left(r_{x}, r_{y}\right)
$$

The CoP can be determined as the intersection between wrench-axis and tool surface (see Eq.(3) \& (4)), as graphically shown in Fig.2-b. Once the CoP is determined, it is possible to analytically compute the geometry of contact, in particular, to carry out a first and a second order analysis:

- tangent plane $\Pi$ (first order analysis): required to split the force $\boldsymbol{F}$ into a normal component and into a tangent component, the former being the normal force and the latter being friction. The normal to the surface is denoted as $\boldsymbol{n}$.

- curvature (second order analysis): required to estimate the contact area once the normal force is known (e.g.

\footnotetext{
${ }^{2}$ Eq.(2) can be seen as a linear system $\boldsymbol{T}=[\boldsymbol{F} \times] \boldsymbol{r}$, where $[\boldsymbol{F} \times]$ is a rank 2 matrix

${ }^{3}$ In general, as shown later, there will be two intersections but only one will have the force $\boldsymbol{F}$ pushing into the mounting-bit
}

based on Hertzian contacts model [20]). The curvature is encoded in terms of maximum and minimum curvatures $\left(\kappa_{\max }, \kappa_{\min }\right)$. The curvature at contact is used in material removal models [31].

As an example, we shall now apply previous equations to a specific mounting-bit: B52 abrasive mounted point from RS components. As for most of the tooling bits, the mountingbit surface $S$ is a surface of revolution and can be determined from the profile. To estimate the surface of the mounting-bit, we used a photographic image of the tool itself and fitted the profile of the tool to an 8-degree polynomial expression using Polyfit function from MATLAB, as shown in Fig.2-a. The equation of the surface can be obtained as a revolution of fitted profile around the $z$-axis. For a point $\boldsymbol{r}=\left[\begin{array}{ll}x y z & z]^{T}\end{array}\right.$ to be on the surface $S$, the height $z$ (z-axis coordinate) is simply a function of its distance $\left(\sqrt{x^{2}+y^{2}}\right)$ from the axis, as follows:

$z=S(x, y)=\sum_{i=1}^{8} p_{i}\left(\sqrt{x^{2}+y^{2}}\right)^{i}$

where the $p_{1}, p_{2}, \ldots, p_{8}$ are the fitting parameters.

The fitted surface represents the actual surface only for points close enough to the central axis $\left(\sqrt{x^{2}+y^{2}} \leq 5.3 \mathrm{~mm}\right)$. Using $x$ and $y$ as parameters, various geometric features of the point of contact can be estimated [31,32]. For any 3D

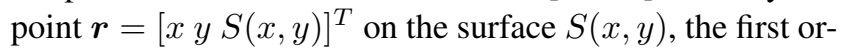
der analysis depends on the vector $\boldsymbol{n}$ normal to the surface and two vectors $\boldsymbol{r}_{x}$ and $\boldsymbol{r}_{y}$ tangent to the surface, which can 


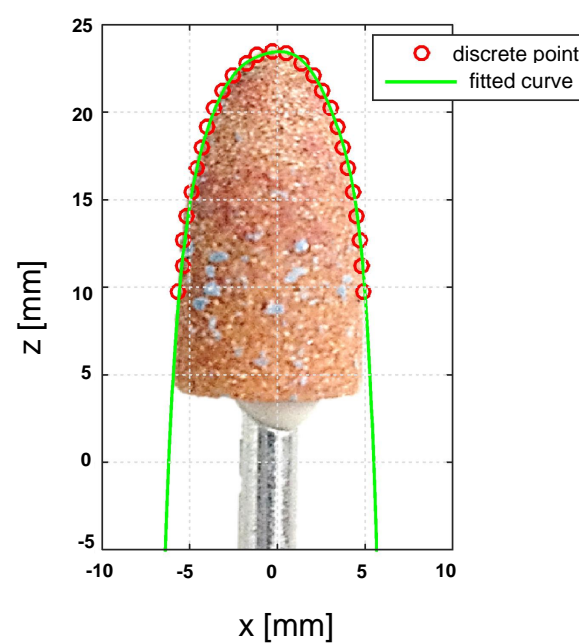

a)

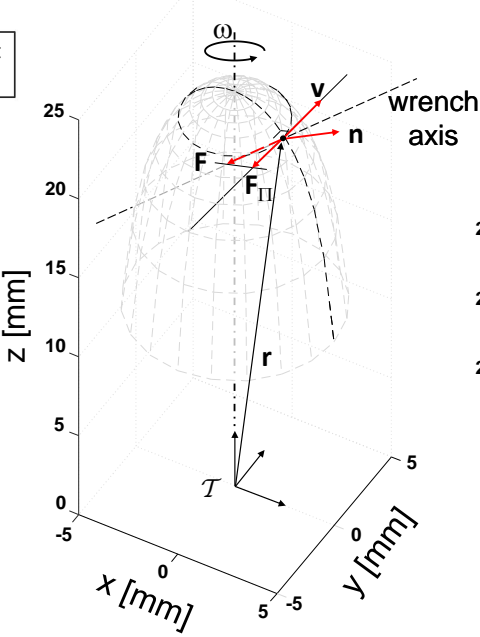

b)

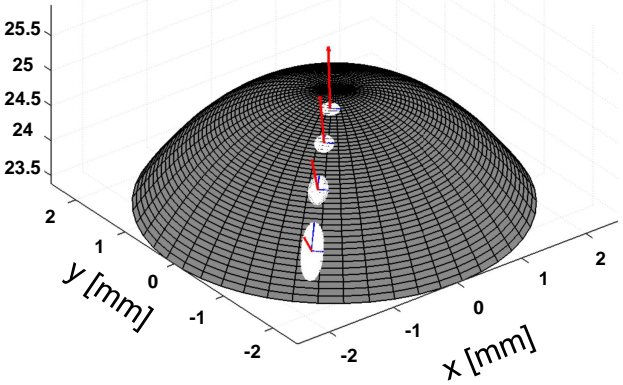

c)

Fig. 2 a) The mounting-bit profile is estimated from a photographic image. b) The CoP is estimated as the intersection between the wrench axis and the surface derived from the mounting-bit profile as a surface of revolution. c) Zoom figure top of the mounting-bit; the normal, tangent vectors, and the Dupin indicatrix (ellipses) to the surface at four different points.

be determined as:

$$
\begin{gathered}
\boldsymbol{r}_{x}:=\frac{\partial \boldsymbol{r}}{\partial x}=\left[\begin{array}{c}
1 \\
0 \\
\frac{\partial S(x, y)}{\partial x}
\end{array}\right] ; \boldsymbol{r}_{y}:=\frac{\partial \boldsymbol{r}}{\partial y}=\left[\begin{array}{c}
0 \\
1 \\
\frac{\partial S(x, y))}{\partial y}
\end{array}\right] \\
\boldsymbol{n}=\frac{\boldsymbol{r}_{x} \times \boldsymbol{r}_{y}}{\left\|\boldsymbol{r}_{x} \times \boldsymbol{r}_{y}\right\|}
\end{gathered}
$$

For a second order analysis, also the second derivatives have to be evaluated:

$$
\begin{gathered}
\boldsymbol{r}_{x x}:=\frac{\partial \boldsymbol{r}_{x}}{\partial x}=\left[\begin{array}{c}
0 \\
0 \\
\frac{\partial^{2} S(x, y)}{\partial x^{2}}
\end{array}\right] ; \boldsymbol{r}_{x y}:=\frac{\partial \boldsymbol{r}_{x}}{\partial y}=\left[\begin{array}{c}
0 \\
0 \\
\frac{\partial^{2} S(x, y)}{\partial x \partial y}
\end{array}\right] \\
\boldsymbol{r}_{y y}:=\frac{\partial \boldsymbol{r}_{y}}{\partial y}=\left[\begin{array}{c}
0 \\
0 \\
\frac{\partial^{2} S(x, y)}{\partial y^{2}}
\end{array}\right]
\end{gathered}
$$

Predictive models of polished surfaces require curvature information at the point of contact $[13,31]$. For this, the coefficients of the first fundamental form $E(x, y):=\boldsymbol{r}_{x}^{T} \cdot \boldsymbol{r}_{x}$, $F(x, y):=\boldsymbol{r}_{x}^{T} \cdot \boldsymbol{r}_{y}$, and $G(x, y):=\boldsymbol{r}_{y}^{T} \cdot \boldsymbol{r}_{y}$ and of the second fundamental form $e(x, y):=\boldsymbol{n}_{p}^{T} \cdot \boldsymbol{r}_{x x}, f(x, y):=\boldsymbol{n}_{p}^{T} \cdot \boldsymbol{r}_{x y}$ and $g(x, y):=\boldsymbol{n}_{p}^{T} \cdot \boldsymbol{r}_{y y}$ should be evaluated. With respect to the tangent frame $\left\{\boldsymbol{r}_{x}, \boldsymbol{r}_{y}\right\}$, it is possible to calculate the so-called Shape Operator matrix $N$ :

$N=\frac{1}{E G-F^{2}}\left[\begin{array}{l}f F-e G e F-f E \\ g F-f G f F-g E\end{array}\right]$

The shape operator matrix can be used, among other things, to determine the so-called Dupin indicatrix, an approximation of the section of the tooling surface $S$ cut by a plane parallel to the tangent plane $\Pi$ at the contact point, and at an infinitesimal distance $\epsilon$.

The Dupin indicatrix also contains important information about the contact area between tool and workpiece which, for elliptic points, i.e. points for which the curvatures have same sign (as in typical mounting bits) is an ellipse [13,31]:

$2 \epsilon=\boldsymbol{w}^{T} N \boldsymbol{w}$

It is possible to solve Eq.(9) for $\boldsymbol{w}=\left[\begin{array}{ll}w_{x} & w_{y}\end{array}\right]^{T}$ and therefore identifying the points belonging to the ellipse's orbit. The vector $\boldsymbol{w}$, that is a $2 \mathrm{D}$ vector of the tangent space at the contact point, can be transformed into a 3D vector by expressing its components with respect to the base $\left\{\boldsymbol{r}_{x}, \boldsymbol{r}_{y}\right\}$

$\boldsymbol{w}=w_{x} \boldsymbol{r}_{x}+w_{y} \boldsymbol{r}_{y}$

The Fig.2-c shows examples of ellipses at four different contact points.

\section{Design of an instrumented hand-held tool}

In this section, we shall describe the instrumentation of a general-purpose, hand-held, rotary tool such as Dremel 4000, shown in Fig.3-a and consisting of a spindle, a flexible shaft and a spinning tool hosting the mounting-bit and hand-held by the operator, see Fig.3-b. The purpose of the flexible shaft is to transmit rotation, generated from a heavy spindle, to the mounting-bit while keeping the tool held by the operator lightweight. 
To be able to capture interaction forces/torques between operator and tool, we designed a 3D printed ergonomic handle for the operator which would be attached to the spinning tool through a force/torque sensor, as shown in Fig.3-b. In the case where the tool is operated by a robot, the ergonomic handle should simply be replaced by a proper attachment jig. To track the tool's motion, a marker-based motion capture system (VZ4000, Poenix Technologies Inc, with a sampling frequency of 100 frames/sec) is used to sense the 3D location of several infra-red markers rigidly attached to the spinning tool.

Although, in theory, only 3 markers are necessary to determine position and orientation of a rigid body, we used a redundant set of 8 markers $\left(m_{1}, m_{2}, \ldots, m_{8}\right)$ to prevent problems such as occlusion, as one or more markers might not be seen by the cameras at some point in time. For this purpose and with reference to Fig.3-c, the optical markers were rigidly attached to two 3D printed brackets used to clamp the spinning tool. A force/torque sensor (ATI loadcell mini 40 , range $F_{X, Y}: \pm 80 N, F_{Z}: \pm 240 N, T_{X, Y Z}$ : $\pm 4 \mathrm{Nm}$ ) is placed between the ergonomic handle and the spinning tool, to capture dynamic interaction (i.e. forces and torques) between operator and spinning tool.

All equations presented in the previous section are written in $\mathcal{T}$ frame coordinate, which is located at the origin of the mounting-bit (i.e. opposite to the tooling end of the mounting-bit). However, since the mounting-bit is typically spinning, having a loadcell directly on the mounting-bit is often impractical. In our case, the loadcell is mounted at a different location, as shown in Fig.3-b, which defines a new (loadcell) reference frame $\mathcal{L}$. As indicated in Fig.3-b, the two main coordinate systems are:

- The tool frame $\mathcal{T}$ : located on the mounting-bit of the spinning tool, with $z$-axis aligned with the major axis and pointing towards the workpiece.

- The loadcell frame $\mathcal{L}$ : the $x$-axis of $\mathcal{L}$ is defined parallel to the $z$-axis of the $\mathcal{T}$ frame, the $z$-axis of $\mathcal{L}$ is defined parallel to an axis of the handle.

In order to estimate the wrench $\boldsymbol{W}^{\mathcal{T}}$ in Eq.(1), in $\mathcal{T}$ frame coordinates, from the wrench $\boldsymbol{W}^{\mathcal{L}}$ as measured directly by the loadcell, the following transformation is used:

$\boldsymbol{W}^{\mathcal{T}}=\left[\begin{array}{cc}R & 0 \\ R \hat{\Delta}^{\mathcal{L}} & R\end{array}\right]\left(\boldsymbol{W}^{\mathcal{L}}-\boldsymbol{W}_{g r}^{\mathcal{L}}\right)$

where the matrix $\hat{\Delta}^{\mathcal{L}}$ and the matrix $R$ represent, respectively, the skew-symmetric matrix of the vector $\Delta^{\mathcal{L}}$ which is the origin of the $\mathcal{T}$ frame expressed in $\mathcal{L}$ coordinates; and the rotation matrix representing the axes of the $\mathcal{T}$ frame in
$\mathcal{L}$ coordinates. In particular, as shown in Fig.3-b:

$$
\begin{array}{cc}
\Delta^{\mathcal{L}}=\left[\begin{array}{c}
\Delta x \\
0 \\
\Delta z
\end{array}\right] ; & \hat{\Delta}^{\mathcal{L}}=\left[\begin{array}{ccc}
0 & -\Delta z & 0 \\
\Delta z & 0 & -\Delta x \\
0 & \Delta x & 0
\end{array}\right] \\
R=\left[\begin{array}{lll}
0 & 1 & 0 \\
0 & 0 & 1 \\
1 & 0 & 0
\end{array}\right]
\end{array}
$$

It should be noted that the wrench $\boldsymbol{W}^{\mathcal{L}}$ contains the readings from the ATI load-cell which, in addition to the interaction forces and torques, will also contain gravitational forces and torques due to the mass of the spinning tool and clamping brackets. The center of mass ( $\boldsymbol{\zeta}$, as in Fig.3-b) of the spinning tool and clamping brackets can be accurately estimated from CAD and the gravitational wrench $\boldsymbol{W}_{g r}^{\mathcal{L}}$ can be determined as:

$\boldsymbol{W}_{g r}^{\mathcal{L}}=m\left[\begin{array}{c}R^{\mathcal{L}} \boldsymbol{g} \\ \boldsymbol{\zeta}^{\mathcal{L}} \times R^{\mathcal{L}} \boldsymbol{g}\end{array}\right]$

where $m$ is the mass of of the spinning tool and backets complex, $R^{\mathcal{L}}$ is the orientation of the $\mathcal{L}$ frame as given by the motion tracker and $\boldsymbol{g}=\left[\begin{array}{lll}0 & 0 & 9.81\end{array}\right]^{T}$ is the gravitational acceleration (in space coordinates, as defined by the motion tracker).

\section{Experimental validation}

This section describes an experimental validation of the instrumented tool and algorithms presented above. In particular, we shall use a flat wooden surface as the workpiece and the hard mounting-bit which is identified clearly in section 2.

A major challenge in tooling operations, with respect to literature, is that we cannot compare our dynamic estimation method directly with kinematic measurements, for example, from a motion tracker, because as the mounting-bit mills through the workpiece's surface, the geometry of the workpiece will change. For this reason, we shall also sensorize the workpiece, by placing a loadcell directly underneath the workpiece, using a 2 -step validation:

i. validation of the dynamic estimation of contact by the instrumented workpiece without mechanical vibrations via simple contact with a rigid and non-rotating stylus. This will allow us to use data from the motion tracker for validation since no abrasive phenomena are taking place.

ii. validation of the dynamic estimation of contact by instrumented tool in the presence of mechanical vibrations, arising during an actual grinding process, by comparison with estimation from the instrumented workpiece. 


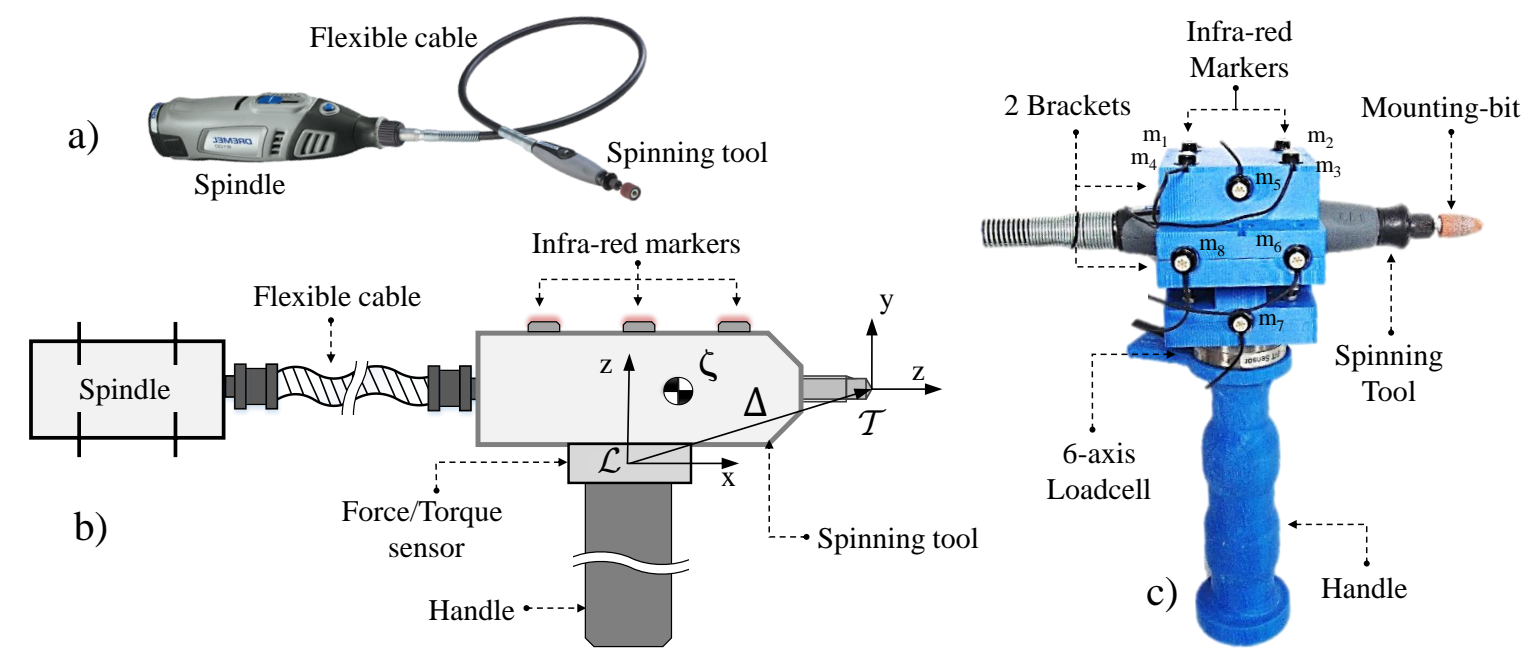

Fig. 3 a) The Dremel tool including spindle, flexible cable and spinning tool. b) The conceptual design. c) The actual instrumented tool.

It should be noted that, in the first validation step, when the loadcell is mounted directly under the workpiece, there will be no 'mechanical noise', e.g. due to vibrations of the tool itself. The accuracy of contact point estimation by the instrumented workpiece is solely limited by the accuracy of the loadcell measurements. A similar loadcell is used for the instrumented tool, in the second validation step. However, in this case, the process will involve a rotating mounting-bit and abrasive phenomena, adding considerable 'mechanical noise' to the measurements of the contact point. This will necessarily lower the accuracy of the estimation method. It is, therefore, plausible to use the instrumented workpiece (calibrated via motion tracker and in the absence of mechanical noise) to calibrate the instrumented tool.

\subsection{Instrumented workpiece}

As described above, the first of the 2-step validation involves sensorizing the workpiece and validating the dynamic estimation of contact in the absence of mechanical vibrations. For this purpose, a loadcell is mounted directly underneath the workpiece, as schematically shown in Fig.4. The workpiece will act as 'touch pad' and, using the method described in Sec. 2, we shall be able to estimate the contact point once a rigid, non-rotating stylus is in contact with the surface. The same equations (3)-(4) hold, where the $\mathcal{T}$ frame is now at the center of the workpiece, see Fig.4. In this specific case, the surface $\mathcal{S}(x, y)$ is the plane of the workpiece, i.e. Eq.(4) is simply $r_{z}=0$, which leads to the following dynamic estimation of contact point

$\boldsymbol{r}^{*}=\left[-\frac{T_{y}}{F_{z}} \frac{T_{x}}{F_{z}} 0\right]^{T}$

At the same time, the point of contact between stylus and surface can be estimated via kinematic measurements de- rived from a set of markers mounted on the stylus itself and on the workpiece.

In a validation experiment, the stylus was manually placed in contact with the workpiece in different quadrants of the workpiece (areas I, II, III, IV, shown in Fig.5-a), with various force levels and varying angles of contact. The accuracy of dynamic estimation in (14) will be reduced at lower values of the signal-to-noise ratio (i.e. when no or little contact force is applied, the measurements from the loadcell are most corrupted by noise). This is apparent in Fig.5-b, where the accuracy improves as the force levels increase. In particular, for forces above $10 \mathrm{~N}$, the estimation error is consistently below $2 \mathrm{~mm}$, which indicates that the used loadcell is reliable for estimating the contact point with the applied force higher than $10 N$.

\subsection{Instrumented tool validation}

The second step of the validation consists of comparing the dynamic estimation of the contact point, as derived from the instrumented tool, with the estimation derived from the instrumented workpiece which was validated in the previous section. For this purpose, the experimental setup shown in Fig. 6 was devised. The setup includes the instrumented tool, as described in Sec.3, and an instrumented workpiece as described in Sec.4.1. Note that the aluminum-frame is connected to the jig via a revolute joint, hence, it is able to slide left/right and achieve any specific angle. The position of the aluminum-frame is tracked by the motion capture system based on the infra-red markers attached on the workpiece. Both the tool and workpiece are instrumented with similar loadcells (respectively, loadcell 1 and loadcell 2 in Fig.6) and with infra-red markers. As shown in Fig.6, three major reference frames shall be considered: 


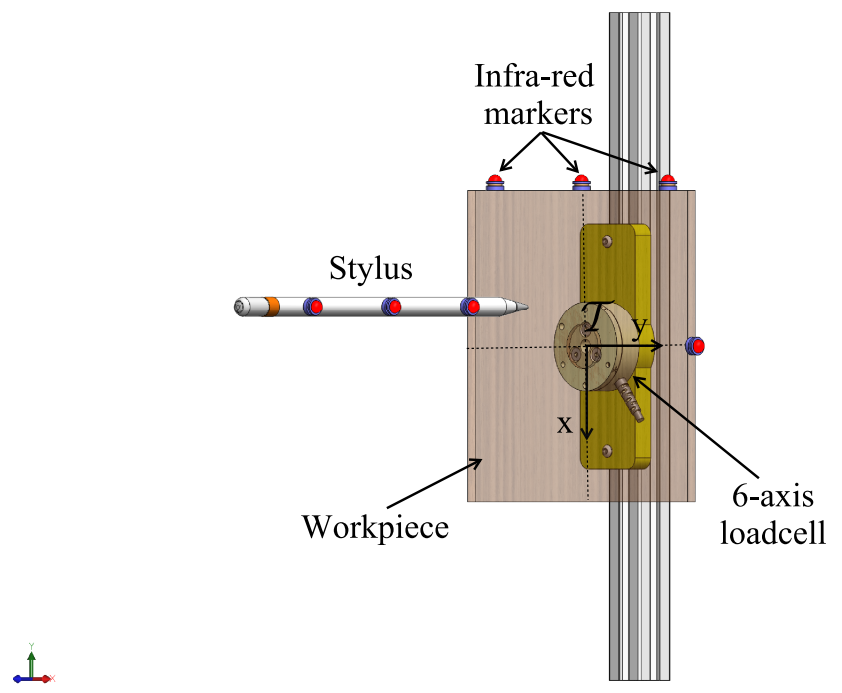

Fig. 4 Calibration Loadcell Setup to compare the contact point measured from motion capture and loadcell: three infra-red markers are attached on the stylus, and a 6-axis loadcell is located under the workpiece.

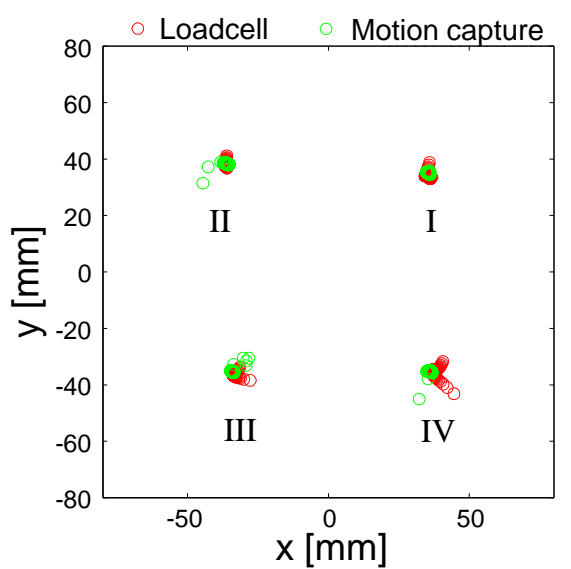

a)

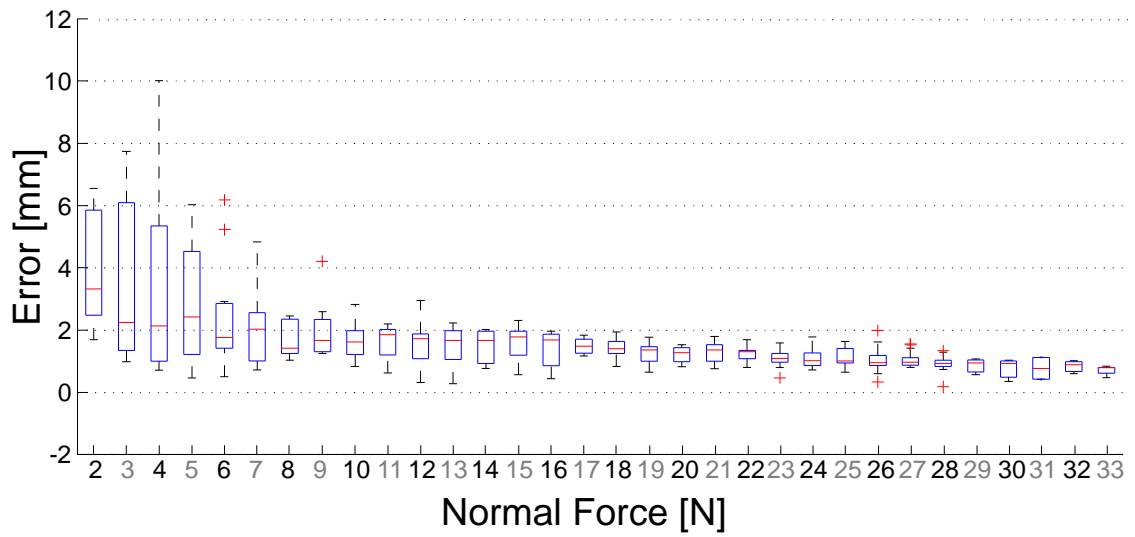

b)

Fig. 5 a) Contact points measured from motion capture and loadcell on the plane's surface. b) Distance error measured from motion capture and loadcell when increasing the force applied on the plane.

- tool frame $\mathcal{T}$ : located at the origin of the rotating mountingbit (note that the frame $\mathcal{T}$ is not rotating but simply remains aligned with the mounting-bit at all times).

- global frame $\mathcal{S}$ : defined by the motion tracker, via external markers mounted on the jig.

- workpiece frame $\mathcal{W}$ : attached to the centre of the workpiece, with the $\mathrm{z}$-axis normal to the workpiece (note that the frame $\mathcal{W}$ can be changed with respect to the frame $\mathcal{S}$ based on the position of the aluminum-frame but the $\mathrm{z}$-axis is always normal to the workpiece).

All the frames are defined by the motion capture system. The instrumented tool is fixed on a base; the workpiece can be manually slid on the jig and tilted to two different inclinations $\left(150^{\circ}, 175^{\circ}\right)$ of the mounting. These various inclinations were chosen to ensure contact points with differ- ent curvature on the mounting-bit. For each of these inclinations, an experimental session was conducted whereby the mounting-bit was brought into contact with the workpiece, set at the rotational speed of 5000 RPM and then the workpiece is slid horizontally (i.e. along the $\mathrm{x}$-axis direction of the $\mathcal{T}$ frame). After each session, a horizontal trace was left on the workpiece due to the abrasive action of the mountingbit. Attention was given to moving the workpiece after each session so that any new trace would occur at a different location from the previous ones. As expected, the width of the trace would vary depending on the inclination of the mounting-bit, in particular due to the different contact point curvatures. When in contact, the mounting-bit will be touching the workpiece a point $\boldsymbol{r}$, which will be simultaneously estimated by the instrumented tool and by the instrumented 


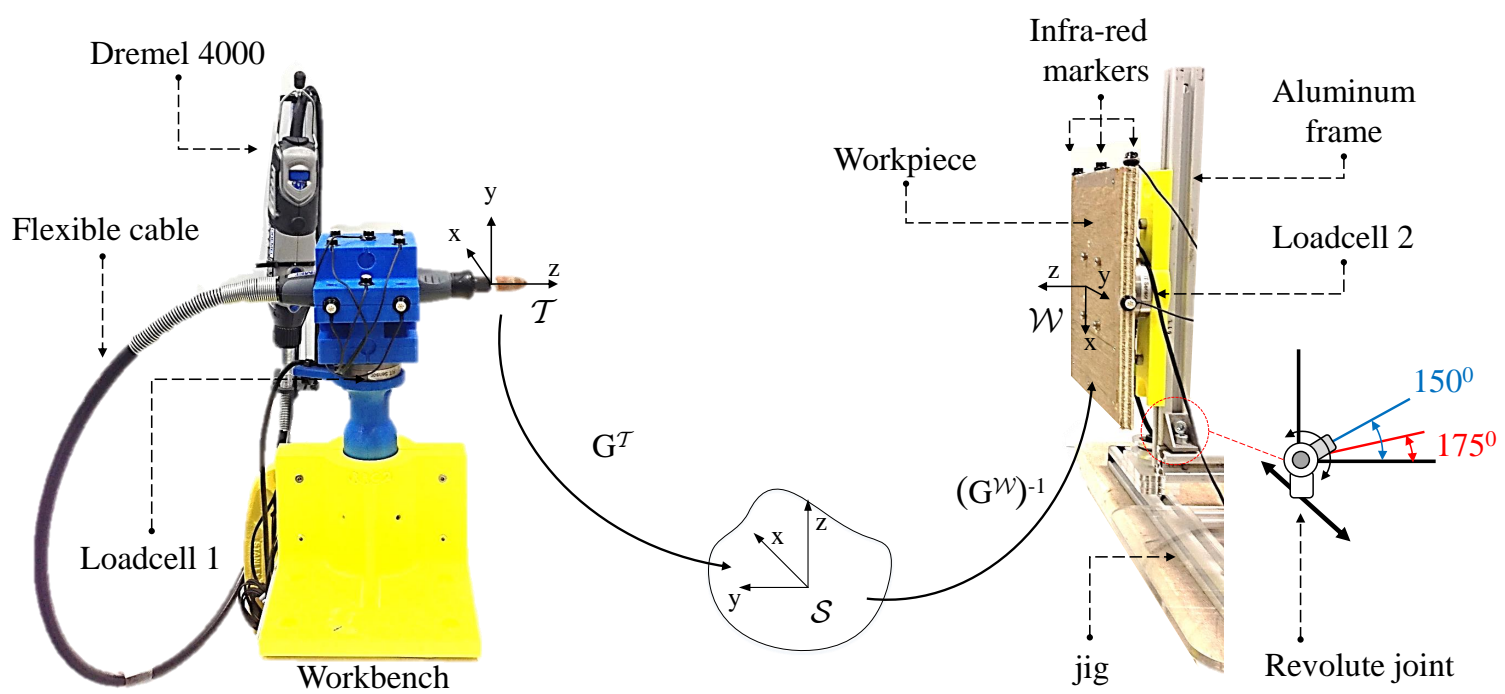

Fig. 6 Experimental setup: the instrumented tool is fixed on a workbench; the workpiece can be manually slid on the jig (i.e. along the x-axis direction of the $\mathcal{T}$ frame) and tilted to two different inclinations $\left(150^{\circ}, 175^{\circ}\right)$ of the mounting. When in touch, the contact point is dynamically estimated by both the tool frame $\mathcal{T}$ and workpiece frame $\mathcal{W}$ coordinate related to one another via the motion tracker transformation.

workpiece. To be compared, the two estimations must be expressed in the same coordinate frame, (e.g. in $\mathcal{W})$. Denoting $\boldsymbol{r}_{1}$ the estimation derived from the instrumented tool (via loadcell 1) via Eqs. (3)-(4) in $\mathcal{T}$ frame and reported in $\mathcal{L}$ coordinates via the following coordinate transformation:

$\left[\begin{array}{c}\boldsymbol{r}_{1} \\ 1\end{array}\right]=\left(\boldsymbol{G}^{\mathcal{W}}\right)^{-1} \boldsymbol{G}^{\mathcal{T}}\left[\begin{array}{c}\boldsymbol{r}^{\mathcal{T}} \\ 1\end{array}\right]$

where $\boldsymbol{G}^{\mathcal{T}}$ and $\boldsymbol{G}^{\mathcal{W}} \in S E(3)$ are homogeneous matrices derived directly from the markers transforming, respectively, the components in $\mathcal{T}$ frame and $\mathcal{W}$ frame into $\mathcal{S}$ frame, (see Fig.6).

The results of the analysis carried out on two experimental tests are shown in Fig.7, for the inclination of $150^{\circ}$ (left panes) and $175^{\circ}$ (right panes). For all plots, the horizontal axis represents the $y$-axis in the workpiece coordinates, i.e. the horizontal direction along which the sliding of the tool occurred. The tool first contacted the workpiece approximately at $y=30 \mathrm{~mm}$ and was then slid until $y=-50 \mathrm{~mm}$.

The first row (i) in Fig.7 shows the contact point coordinates, during the whole experiment, as estimated from the sensors on the tool (dashed line) and from the workpiece (solid line). In this case, the estimation from the workpiece is considered more accurate, or less affected by mechanical vibrations. Previous experiments in Fig.5 reports that the estimations from the workpiece sensors are subject to less than $2 \mathrm{~mm}$ errors when the force is higher than $10 \mathrm{~N}$. This information can be found in the second row (ii) of Fig.7 where both the contact error and the force magnitude (for both normal force and friction components, i.e. the projection along the normal to the mounting-bit surface and the projection on the workpiece tangent to the surface at contact) are reported.
It is possible to notice that the error increases when the contact force decreases.

The third row (iii) of Fig.7, shows the magnitude and direction of friction at different contact points. Estimations from both the workpiece and mounting-bit are reported. It is clear that the friction forces as sensed by the workpiece are pointing in oppositite direction with respect to the friction forces as sensed by the mounting-bit.

Finally, the bottom row (iv) of Fig.7 plots the estimated power due to friction. In the hypothesis of a sufficiently localized contact, one can assume that the velocity of all abrasive grains on the mounting-bit has the same tangential velocity (as a result of tool rotation $\omega$, in our case 5000 RPM). The tangential velocity $\boldsymbol{v}$ can then be estimated as

$\boldsymbol{v}=\omega \boldsymbol{r} \times\left[\begin{array}{lll}0 & 0 & 1\end{array}\right]^{T}$

and the power PWR can then be estimated as

$P W R=\boldsymbol{F} \cdot \boldsymbol{v}$

Finally, Fig. 8 shows the abrasive action of the mountingbit on the workpiece. In particular, Fig.8-a depicts a photograph of the workpiece after the abrasive action with superimposed estimated contact points and computed contact ellipses. It can be seen that: 1) the estimated contact line (green line) matches with the trace on the wood-workpiece; 2) the width of trace, which depends on the contact area between the mounting-bit and the wood-workpiece, is well captured by the area of the estimated ellipses. Fig.8-b shows a 3D scan obtained from an ATOS Triple Scan camera to visualize the depth of the $150^{\circ}$ trace. This illustrates how the contact area can be visualized and the information used to evaluate the dimension of the contact ellipses. 
$150^{\circ}$

i)

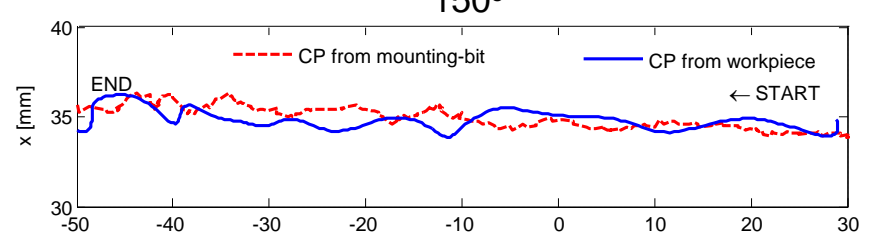

ii)
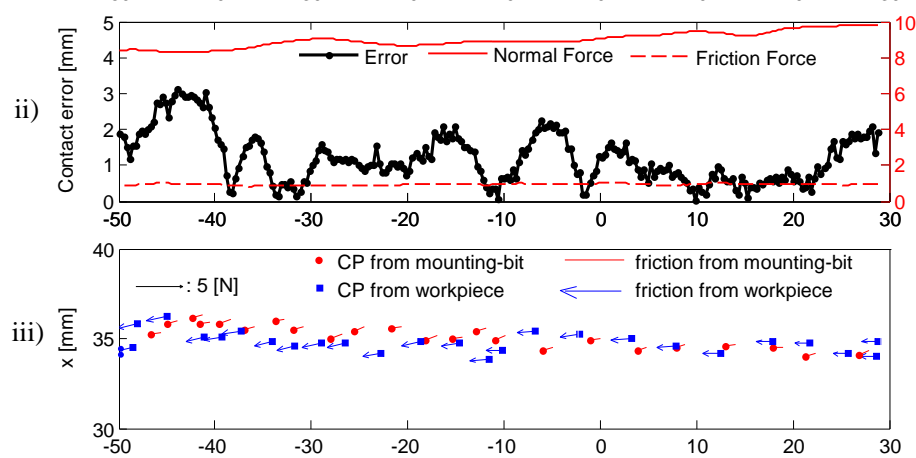

iv)

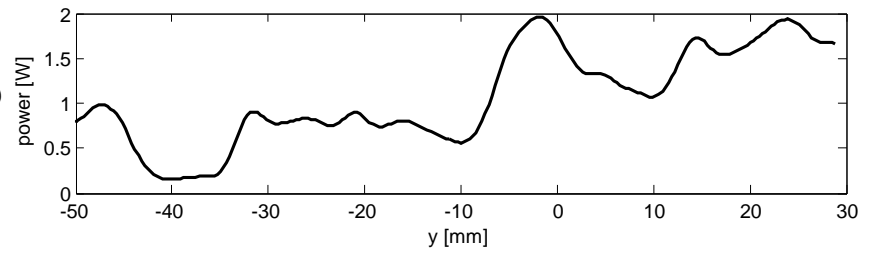

a) $175^{\circ}$
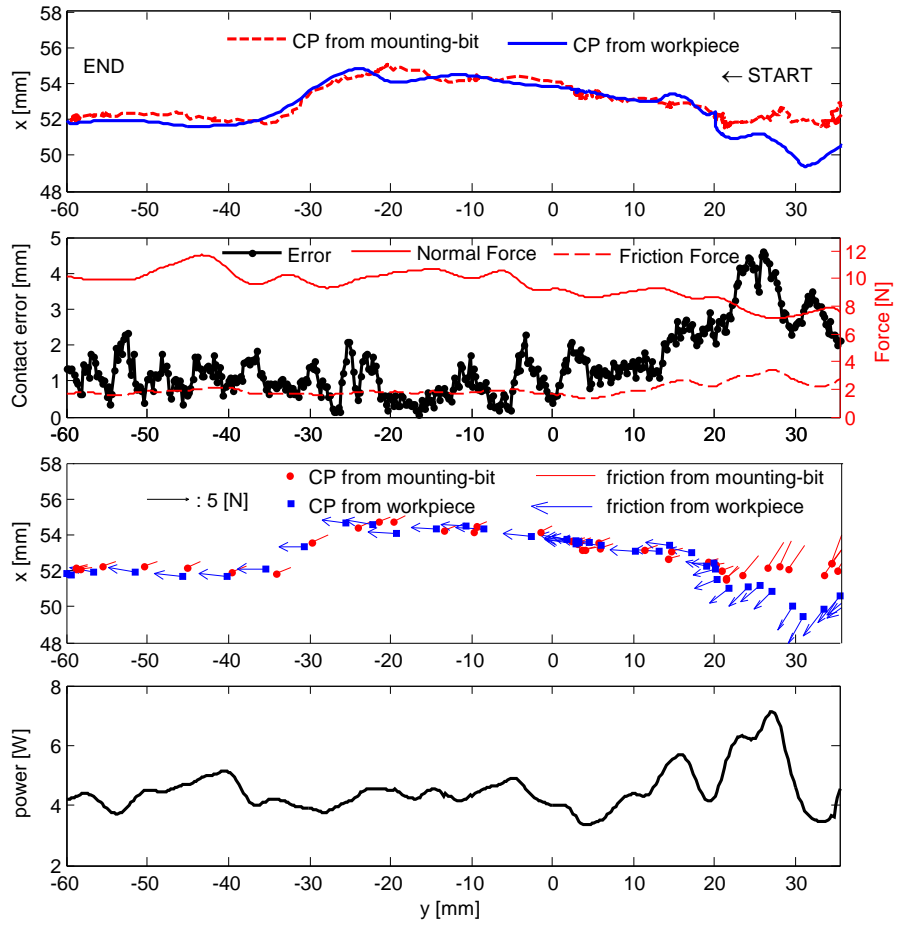

b)

Fig. 7 i) Contact points measured from mounting-bit and from the workpiece, on the workpiece space $\mathcal{W}$. ii) Error between contact points from tool and from workpiece with respect to the applied normal force. iii) The estimated friction force from the mounting-bit from the workpiece. iv) The estimated power due to friction and contact points.

\section{CONCLUSION and DISCUSSION}

This study presents a framework which allows capturing 3D interaction dynamics, 'dynamic touch', during manual operation tasks. In particular, we introduce the design of an instrumented hand-held power tool capable of online sensing, suitable to both manual and robotic operations.

Online-sensing could improve traditional methods to determine suitable tool paths as they often rely on trial and error methods. While the expertise of robot programmers is of utterly importance [33], capturing 3D interaction dynamics could improve robotic polishing especially when the surface and its geometry change continuously as the unwanted material is removed to produce the desired initial workpiece shape [16,34].

Algorithms to carry out dynamic estimation of contact are adapted from literature and presented in the context of power tools, specifically considering the profile of hard mounting bits. From the estimation of the contact point, first and second order geometric analysis is conducted 1) to be able to split the sensed force into normal and friction components; 2) to estimate the curvatures of the mounting-bit and workpiece at contact. This type of information is critical for ma- terial removal estimates, although the evaluation of MRR is beyond the scope of this work.

The instrumented tool and the algorithms necessary to estimate 'dynamic touch' presented in this paper are a first step towards automating finishing operations by implementing human-like strategies [35]. Outcomes of the study are promising and, the proposed instrumented tool and algorithms can be used to measure the differences between the manual polishing task and the robot-mounted polishing task. Therefore, the number of trials of robot strategy can be reduced to generate the finalized tool path [36]. Moreover, although the proposed algorithms were tested on data derived from manual operations, they can also be readily implemented in robotic applications and used to facilitate tool path programming, e.g. programming by demonstration. The operator can demonstrate the trajectory to the robot by performing the polishing task; the information will then be extracted and the robot is finally planned $[37,38]$.

\section{ACKNOWLEDGMENT}

The work was supported by the A*STAR Industrial Robotics Program, 2013-2016. 
a)

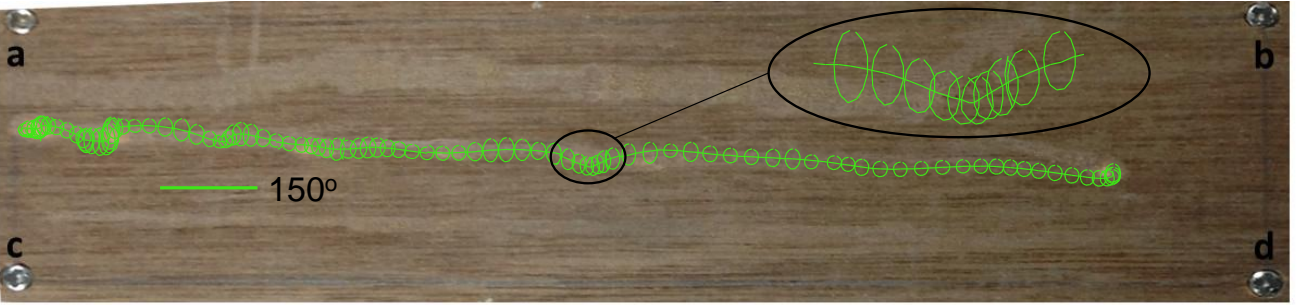

b)

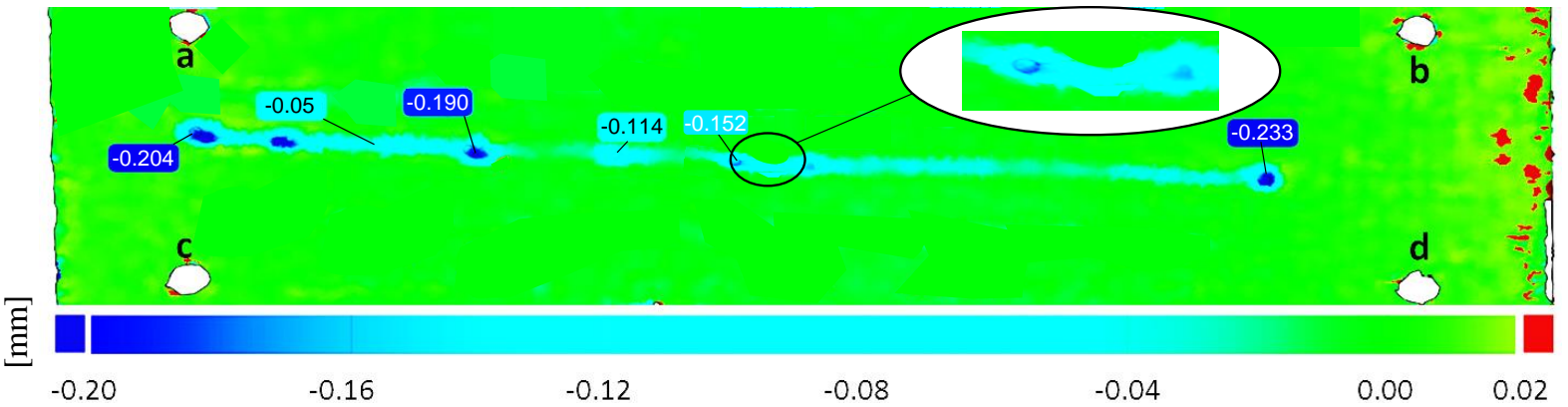

Fig. 8 The workpiece after the grinding process a) The projection of the computed ellipses onto the workpiece $\left(150^{0}\right)$. b) The depth of the contact area after the grinding is represented as a GOM picture.

\section{References}

1. P. Kala and P. M. Pandey, Comparison of finishing characteristics of two paramagnetic materials using double disc magnetic abrasive finishing, Journal of Manufacturing Processes, vol. 17, pp. 63 77, 2015

2. S. Calinon, Robot programming by demonstration. EPFL Press, 2009

3. G. Ganesh, N. Jarrasse, S. Haddadin, A. Albu-Schaeffer, and E. Burdet, A versatile biomimetic controller for contact tooling and haptic exploration, in Robotics and Automation (ICRA), 2012 IEEE International Conference on, pp. 33293334, IEEE, 2012.

4. Y. Li, N. Jarrasse, and E. Burdet, Versatile interaction control and haptic identification in humans and robots, in Geometric and $\mathrm{Nu}-$ merical Foundations of Movements, pp. 187206, Springer, 2017

5. E. Pignat and S. Calinon, Learning adaptive dressing assistance from human demonstration, Robotics and Autonomous Systems, vol. 93, pp. 6175, 2017

6. P. Kormushev, D. N. Nenchev, S. Calinon, and D. G. Caldwell, Upper-body kinesthetic teaching of a free-standing humanoid robot, in Robotics and Automation (ICRA), 2011 IEEE International Conference on, pp. 39703975, IEEE, 2011

7. P. Kormushev, S. Calinon, and D. G. Caldwell, Imitation learning of positional and force skills demonstrated via kinesthetic teaching and haptic input, Advanced Robotics, vol. 25, no. 5, pp. 581603, 2011

8. A. Irish, I. Mantegh, and F. Janabi-Sharifi, A pbd approach for learning pseudo-periodic robot trajectories over curved surfaces, in Advanced Intelligent Mechatronics (AIM), 2010 IEEE/ASME International Conference on, pp. 14251432, IEEE, 2010

9. H. Huang, L. Zhou, X. Chen, and Z. Gong, Smart robotic system for $3 \mathrm{~d}$ profile turbine vane airfoil repair, The International Journal of Advanced Manufacturing Technology, vol. 21, no. 4, pp. 275283 , 2003

10. L. Zhou and H. Huang, An automated robotic system for jet engine overhaul. system design and development for honeycomb repair, The International Journal of Advanced Manufacturing Technology, vol. 19, no. 5, pp. 370376, 2002

11. S. Wu, K. Kazerounian, Z. Gan, and Y. Sun, A simulation platform for optimal selection of robotic belt grinding system parameters, The International Journal of Advanced Manufacturing Technology, pp. 112,2013
12. X. Ren and B. Kuhlenkotter, Real-time simulation and visualization of robotic belt grinding processes, The International Journal of Advanced Manufacturing Technology, vol. 35, no. 11, pp. 10901099 , 2008

13. C. Fan, J. Zhao, L. Zhang, G. S. Hong, Y. S. Wong, and J. Zhao, Predictive models of the local and the global polished profiles in deterministic polishing of free-form surfaces, Proceedings of the Institution of Mechanical Engineers, Part B: Journal of Engineering Manufacture, vol. 228, no. 8, pp. 868879, 2014

14. J. Qi, D. Zhang, S. Li, and B. Chen, A micro-model of the material removal depth for the polishing process, The International Journal of Advanced Manufacturing Technology, pp. 112, 2016

15. D. Feng, Y. Sun, and H. Du, Investigations on the automatic precision polishing of curved surfaces using a five-axis machining centre, The International Journal of Advanced Manufacturing Technology, vol. 72, no. 9-12, pp. 16251637, 2014

16. M. Rososhansky, F. Xi, and Y. Li, Coverage based tool path planning for automated polishing using contact stress theory, in Automation Science and Engineering (CASE), 2010 IEEE Conference on, pp. 592597, IEEE, 2010

17. L. Feng-yun and L. Tian-sheng, Development of a robot system for complex surfaces polishing based on cl data, The International Journal of Advanced Manufacturing Technology, vol. 26, no. 9, pp. 11321137,2005

18. S. Sugita, T. Itaya, and Y. Takeuchi, Development of robot teaching support devices to automate deburring and finishing works in casting, The International Journal of Advanced Manufacturing Technology, vol. 23, no. 3-4, pp. 183189, 2004

19. M. J. Tsai, J.-L. Chang, and J.-F. Haung, Development of an automatic mold polishing system, IEEE Transactions on automation science and engineering, vol. 2, no. 4, pp. 393397, 2005

20. H. Hertz, On the contact of elastic bodies, Journal fur die reine und angewandte Mathematic, vol. 92, p. 156-171. (In German.), 1881

21. J. K. Salisbury Jr, Interpretation of contact geometries from force measurements, in Robotics and Automation. Proceedings. 1984 IEEE International Conference on, vol. 1, pp. 240247, IEEE, 1984

22. D. Brock, Environment perception of an articulated robot hand using contact sensors, Robotics and Manufacturing Automation, vol. 15 , pp. 8996,1985

23. T. Tsujimura and T. Yabuta, Object detection by tactile sensing method employing force/torque information, Robotics and Automation, IEEE Transactions on, vol. 5, no. 4, pp. 444450, 1989 
24. B. S. Eberman and J. K. Salisbury, Determination of manipulator contact information from joint torque measurements, in Experimental Robotics I, pp. 463473, Springer, 1990

25. A. Bicchi, J. K. Salisbury, and D. L. Brock, Contact sensing from force measurements, The International Journal of Robotics Research, vol. 12 , no. 3 , pp. 249262,1993

26. J. Salisbury, Kinematic and force analysis of articulated hands, department of computer science stanford university report no, STAN CS, vol. 89, p. 921,1982

27. K. H. Hunt, Kinematic geometry of mechanisms. Clarendon Press Oxford, 1990

28. X. Zhou, Q. Shi, and Z. Li, Contact localization using force/torque measurements, in Robotics and Automation, 1996. Proceedings., 1996 IEEE International Conference on, vol. 2, pp. 13391344, IEEE, 1996

29. L. Cen and S. N. Melkote, Effect of robot dynamics on the machining forces in robotic milling, Procedia Manufacturing, vol. 10, pp. 486496,2017

30. M.-J. Tsai, J. Huang, and W. Kao, Robotic polishing of precision molds with uniform material removal control, International Journal of Machine Tools and Manufacture, vol. 49, no. 11, pp. 885895, 2009

31. S. P. Radzevich, Kinematic geometry of surface machining. CRC Press, 2007

32. D. Carmo and M. Perdigao, Differential geometry of curves and surfaces, vol. 2. Prentice-hall Englewood Cliffs, 1976

33. G. F. Rossano, C. Martinez, M. Hedelind, S. Murphy, and T. A. Fuhlbrigge, Easy robot programming concepts: An industrial perspective, in Automation Science and Engineering (CASE), 2013 IEEE International Conference on, pp. 11191126, IEEE, 2013

34. W. Wei and Y. Chao, A path planning method for robotic belt surface grinding, Chinese journal of aeronautics, vol. 24, no. 4, pp. 520526, 2011

35. A. Zanchettin, L. Bascetta, and P. Rocco, Human-like redundancy resolution for anthropomorphic industrial manipulators, IEEE Robotics \& Automation Magazine, 2012

36. W. Ng, H. Chan, W. Teo, and I. Chen, Capturing the tacit knowledge of the skilled operator to program tool paths and tool orientations for robot belt grinding, The International Journal of Advanced Manufacturing Technology, vol. 91, no. 5-8, pp. 15991618, 2017

37. S. Calinon and A. Billard, Recognition and reproduction of gestures using a probabilistic framework combining pca, ica and hmm, in Proceedings of the 22nd international conference on Machine learning, pp. 105112, ACM, 2005

38. A. Klimchik, A. Ambiehl, S. Garnier, B. Furet, and A. Pashkevich, Experimental study of robotic-based machining, IFACPapersOnLine, vol. 49, no. 12, pp. 174179, 2016 